\title{
Mesonic spectral functions in effective mean field model.
}

\section{Chowdhury Aminul Islam*}

Theory Division, Saha Institute of Nuclear Physics, Kolkata

E-mail: chowdhury . aminulislamegmail.com

\begin{abstract}
We have studied the vector meson spectral function (VMSF) in a hot and dense medium within an effective QCD model namely the Nambu-Jona-Lasinio (NJL) and its Polyakov Loop extended version (PNJL) with and without the effect of isoscalar vector interaction (IVI). The effect of the IVI has been taken into account using the ring approximation. We obtained the dilepton production rate (DPR) using the VMSF and observed that at moderate temperature it is enhanced in the PNJL model as compared to the NJL and Born rate due to the suppression of color degrees of freedom. We also computed the conserved density fluctuation associated with the temporal correlation function appropriate for a hot and dense medium.
\end{abstract}

7th International Conference on Physics and Astrophysics of Quark Gluon Plasma

1-5 February, 2015

Kolkata, India

*Speaker. 


\section{Introduction}

Under extreme conditions, i.e., at high temperature and/or high density Quantum Chromodynamics $(\mathrm{QCD})$ exhibits a very rich and interesting phase structure. It is believed that at these extreme conditions normal hadronic matter transforms into a deconfined state of strongly correlated quark gluon plasma (QGP) [1,2] . A lot of efforts have been put to create and explore this noble state of matter in laboratory through experiments of heavy ion collisions such as Relativistic Heavy Ion Collider (RHIC) at Brookhaven National Lab (BNL) [3, 4] and the Large Hadron Collider (LHC) at the European Organization for Nuclear Research (CERN) [5, 6]. There is also an upcoming fixed target experiment named as Facility for Antiproton and Ion Research (FAIR) at the Gesellschaft für Schwerionenforschung (GSI)[7]. Various measurements at RHIC BNL [8] and LHC CERN [9] have indicated a strong hint of the creation of a strongly correlated QGP [10].

Many properties of the deconfined, strongly interacting matter are reflected in the structure of the correlation function (CF) and its spectral representation [11]. The temporal part of the CF reflects response of the conserved density fluctuations whereas the spatial part reveals the information on the masses and width. We can construct mesonic current-current CF's from meson currents and they can be of different types such as scalar, pseudoscalar, vector and pseudovector [12]. The spectral representation of vector meson current-current $\mathrm{CF}$ is related to the differential lepton pair production rate [13]. These leptons, being weakly interacting, carry informations about the earlier stage of the produced QGP. Keeping this in mind we construct the vector meson spectral function (VMSF) and obtain the dilepton production rate (DPR) from it and also calculate quark number susceptibility (QNS) associated with the temporal component of the VMSF through the conserved density fluctuation (CDF).

Now there are many theoretical tools which can be used to study these CF's and their spectral representations - as for example first principle calculation like Lattice QCD (LQCD) [14], perturbative calculation known as perturbative QCD (PQCD) $[15,16,17]$ and effective QCD models $[18,19]$. These theoretical tools have their own merits and demerits. This present work is based on the effective QCD model namely the Nambu-Jona-Lasinio (NJL) and its Polyakov Loop extended version (PNJL). We have studied these models with and without the effects of isoscalar vector interaction (IVI). The effect of IVI has been taken into account through ring approximation. In Sec. 2 we briefly introduce the mathematical formalism. The results are discussed in Sec. 3 and in Sec. 4 we conclude.

\section{Theoretical Framework}

The differential DPR is related to the VMSF, $\sigma_{V}$, as [20]

$$
\frac{d R}{d^{4} x d^{4} Q}=\frac{5 \alpha^{2}}{54 \pi^{2}} \frac{1}{M^{2}} \frac{1}{e^{\omega / T}-1} \sigma_{V}(\omega, \vec{q})
$$

where $\alpha$ is the fine structure constant, $Q \equiv\left(q_{0}=\omega, \vec{q}\right)$ is the external four momentum and $M=\sqrt{\omega^{2}-q^{2}}$ is the invariant mass of the lepton pair. 
The QNS, $\chi_{q}$ measures the response of the quark number density $\rho$ with infinitesimal change in the quark chemical potential, $\mu+\delta \mu$ and is given by

$$
\chi_{q}(T)=\left.\frac{\partial \rho}{\partial \mu}\right|_{\mu=0}=\int d^{4} x\left\langle J_{0}(0, \vec{x}) J_{0}(0, \overrightarrow{0})\right\rangle=\lim _{\vec{q} \rightarrow 0} \beta \int \frac{d \omega}{2} \frac{-2}{1-e^{-\omega / T}} \sigma_{00}(\omega, \vec{q})
$$

where $\sigma_{00}$ is the temporal component of the VMSF.

\section{Results}

Here we briefly discuss our findings. The details can be found in Ref.[21]. In Fig. 1 the scaled spectral functions (SF) without the IVI are shown. At $T=200 \mathrm{MeV}$ we observe that the PNJL SF has a larger threshold than the NJL one because of the larger quark mass. This effective quark mass decreases as we increase the temperature and that is why at $T=300 \mathrm{MeV}$ the thresholds for NJL and PNJL are almost the same. We also observe that for the PNJL case the SF has been enhanced. This can be understood with the following argument. For zero external momentum $(q)$ and zero chemical potential $(\mu)$ the SF is proportional to $\left[1-2 f\left(E_{p}\right)\right]$, where $f\left(E_{p}\right)$ is the fermion distribution function. Now the presence of the Polyakov Loop (PL) fields suppress the color degrees of freedom, or in other words suppress $f\left(E_{p}\right)$ and thus enhances the SF.
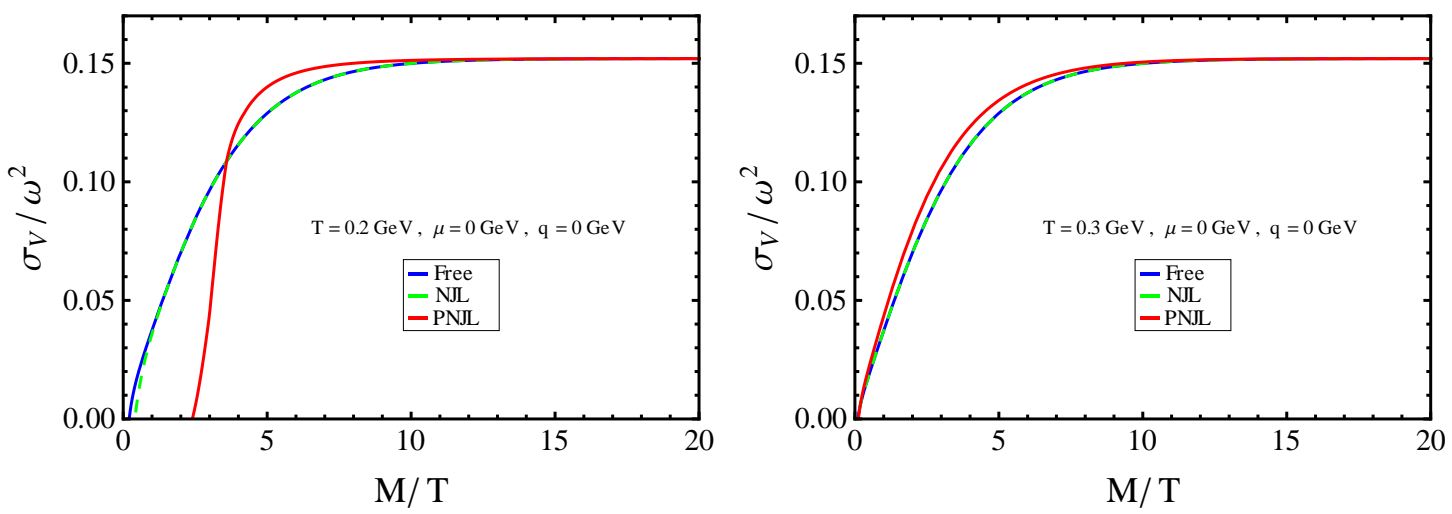

Figure 1: Scaled vector spectral function $\sigma_{V} / \omega^{2}$ as a function of scaled invariant mass, $M / T$ with $q=\mu=0$ and $G_{V} / G_{S}=0$ for $T=200 \mathrm{MeV}$ (left panel) and $T=300 \mathrm{MeV}$ (right panel).

Once we have the SF, the dilepton rate can be calculated from it using Eq. 2.1. The differential dilepton rates are shown in Fig. 2. We notice that the dilepton rate for PNJL model is increased as compared to the NJL and Born rate. We have further compared our findings with the available quenched LQCD results[14].

The SF's with the inclusion of IVI are shown in Fig. 3. It is clear that in the presence of IVI, bound states start to form. The spectral strength increases as we increase the strength of the IVI ${ }^{1}$ and for a given $G_{V}$ the spectral strength for the PNJL is greater than NJL, which is again attributed to the presence of PL fields.

\footnotetext{
${ }^{1}$ The strength of the IVI is represented by $G_{V}$ in terms of $G_{S}$, where $G_{S}$ and $G_{V}$ denote coupling constants of the scalar and vector type four-quark interactions respectively.
} 


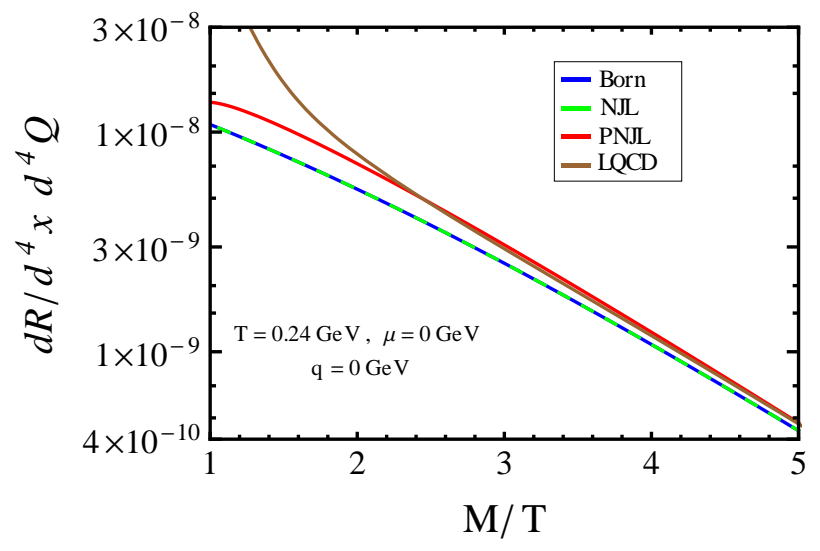

Figure 2: Comparison of dilepton rates as a function of scaled invariant mass $M / T$ for $T=240 \mathrm{MeV}$ with external momentum $q=0$, quark chemical potential $\mu=0$ and $G_{V} / G_{S}=0$. The LQCD rate is from Ref.[14].
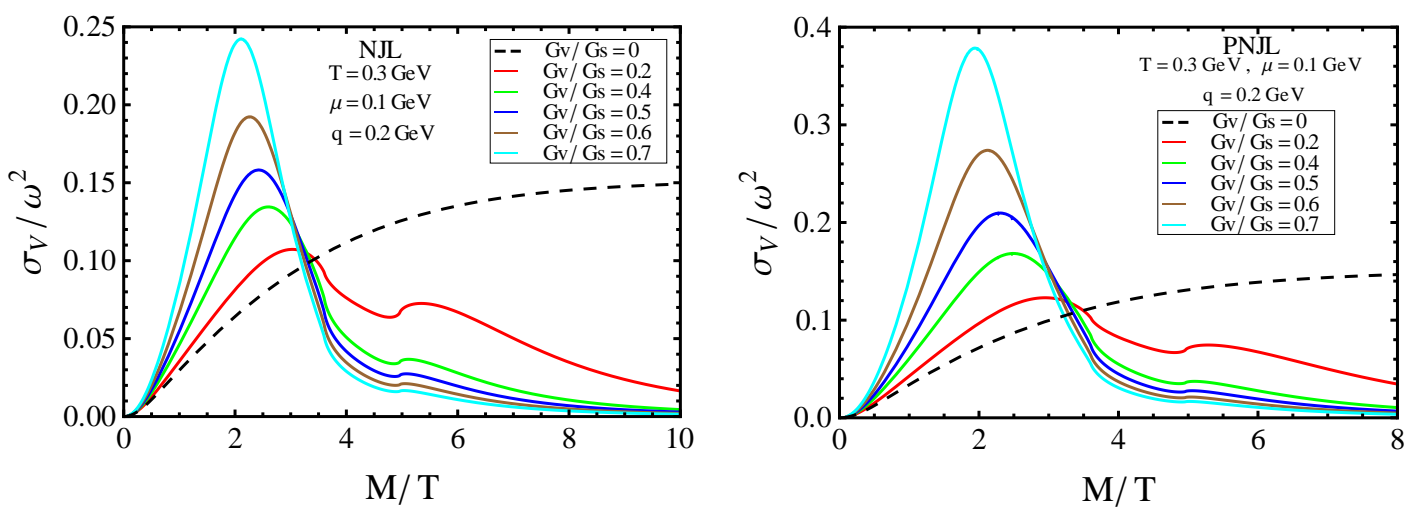

Figure 3: Scaled spectral function as a function of $M / T$ for $T=300 \mathrm{MeV}, \mu=100 \mathrm{MeV}, q=200 \mathrm{MeV}$ and a set of values of $G_{V} / G_{S}$; NJL (left panel) and PNJL (right panel).

In Fig. 4 the differential dilepton rates are shown for both NJL and PNJL models. The dilepton rates increase as the strength of the IVI increases which was expected if we look back at the corresponding plots of spectral function in Fig. 3.

In Fig. 5 the QNS are shown for both NJL and PNJL models. The resummed susceptibility gets suppressed as one increases $G_{V}$. Since positive $G_{V}$ implies a repulsive interaction, the compressibility of the system decreases with the increase of $G_{V}$, hence the suppression.

\section{Summary}

We have investigated the behavior of the VMSF and its spectral representation in a hot and dense medium. The differential dilepton rate has been calculated from the SF for both with and without the IVI. We observed that without the IVI the SF's in NJL become quantitatively equal to those of free field theory. For PNJL they are enhanced as compared to both the NJL and the free field theory. This enhancement is reflected in the corresponding plots of dilepton rates. Suppression of the color degrees of freedom due to the presence of PL fields is the reason behind this enhancement. This suggests that some nontrivial correlation exists among the color charges in the deconfined phase. Thus it is expected that the dilepton rate in a strongly coupled QGP is 

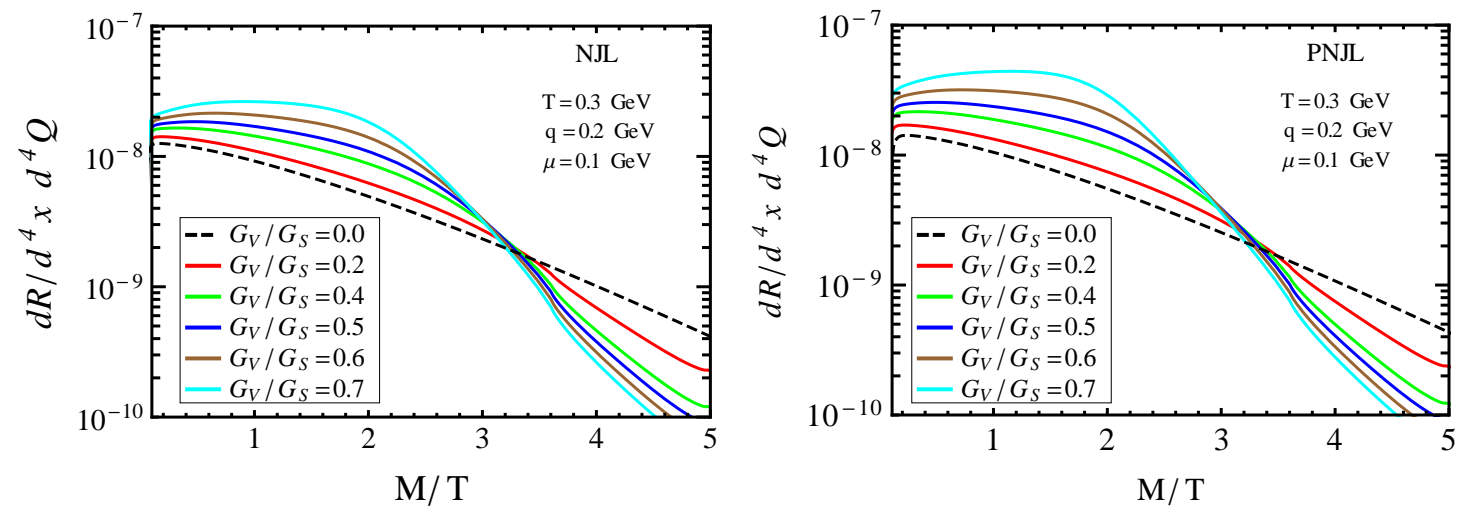

Figure 4: Dilepton rates as a function of $M / T$ for a set of values of $G_{V} / G_{S}$ with $T=300 \mathrm{MeV}, q=200$ $\mathrm{MeV}$ and $\mu=100 \mathrm{MeV}$; NJL (left panel) and PNJL (right panel).
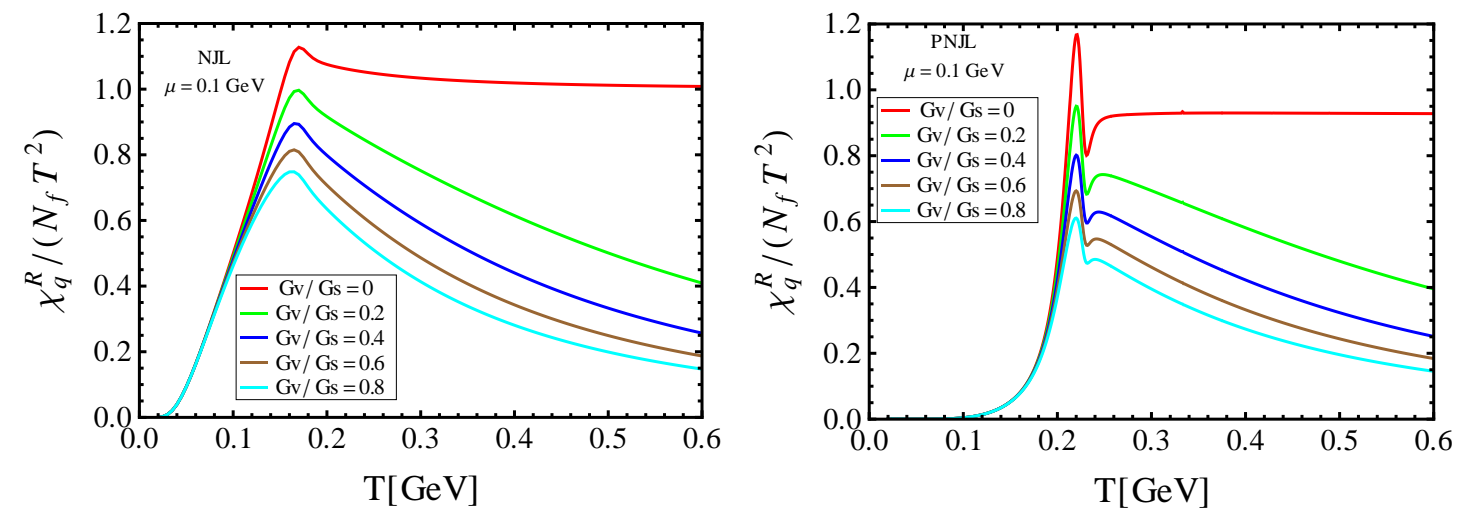

Figure 5: Resummed quark number susceptibility in (a) NJL and (b) PNJL models at non-zero chemical potential for two flavor $\left(N_{f}=2\right)$.

more than that in a weakly coupled one. We have compared some of our results with the available quenched LQCD data and the other results can be tested in future when LQCD includes the dynamical fermions.

\section{Acknowledgement}

CAI was supported financially by the University Grants Commission, India.

\section{References}

[1] B. Muller, “The Physics Of The Quark - Gluon Plasma,” Lect. Notes Phys. 225, 1 (1985).

[2] U. W. Heinz and M. Jacob, "Evidence for a new state of matter: An Assessment of the results from the CERN lead beam program,” nucl-th/0002042.

[3] J. Adams et al. [STAR Collaboration], "Experimental and theoretical challenges in the search for the quark gluon plasma: The STAR Collaboration's critical assessment of the evidence from RHIC collisions," Nucl. Phys. A 757, 102 (2005) [nucl-ex/0501009]. 
[4] K. Adcox et al. [PHENIX Collaboration], "Formation of dense partonic matter in relativistic nucleus-nucleus collisions at RHIC: Experimental evaluation by the PHENIX collaboration," Nucl. Phys. A 757, 184 (2005) [nucl-ex/0410003].

[5] F. Carminati et al. [ALICE Collaboration], “ALICE: Physics performance report, volume I," J. Phys. G 30, 1517 (2004).

[6] B. Alessandro et al. [ALICE Collaboration], “ALICE: Physics performance report, volume II,” J. Phys. G 32, 1295 (2006).

[7] B. Friman, C. Hohne, J. Knoll, S. Leupold, J. Randrup, R. Rapp and P. Senger, "The CBM physics book: Compressed baryonic matter in laboratory experiments," Lect. Notes Phys. 814, 1 (2011).

[8] A. Adare et al. [PHENIX Collaboration], "Scaling properties of azimuthal anisotropy in Au+Au and $\mathrm{Cu}+\mathrm{Cu}$ collisions at $\sqrt{s_{N N}}=200-\mathrm{GeV}$," Phys. Rev. Lett. 98, 162301 (2007) [nucl-ex/0608033].

[9] K. Aamodt et al. [ALICE Collaboration], "Elliptic flow of charged particles in $\mathrm{Pb}-\mathrm{Pb}$ collisions at 2.76 TeV,’ Phys. Rev. Lett. 105, 252302 (2010) [arXiv:1011.3914 [nucl-ex]].

[10] C. A. Islam, R. Abir, M. G. Mustafa, R. Ray and S. K. Ghosh, "The consequences of SU(3) colorsingletness, Polyakov Loop and Z(3) symmetry on a quarkâĂŞgluon gas,” J. Phys. G 41, 025001 (2014) [arXiv:1208.3146 [hep-ph]].

[11] D. Forster, Hydrodynamics Fluctuation, Broken Symmetry and Correlation Function (Benjamin/Cummings, Menlo Park,CA, 1975).

[12] R. M. Davidson and E. Ruiz Arriola, "Mesonic correlation functions in the NJL model with vector mesons,” Phys. Lett. B 359, 273 (1995).

[13] J. I. Kapusta and C. Gale, Finite Temperature Field Theory Principle and Applications (Cambridge University Press, Cambridge, 1996), 2nd ed.

[14] H.-T. Ding, A. Francis, O. Kaczmarek, F. Karsch, E. Laermann and W. Soeldner, “Thermal dilepton rate and electrical conductivity: An analysis of vector current correlation functions in quenched lattice QCD,” Phys. Rev. D 83, 034504 (2011) [arXiv:1012.4963 [hep-lat]].

[15] E. Braaten, R. D. Pisarski and T. C. Yuan, "Production of Soft Dileptons in the Quark - Gluon Plasma," Phys. Rev. Lett. 64, 2242 (1990).

[16] C. Greiner, N. Haque, M. G. Mustafa and M. H. Thoma, "Low Mass Dilepton Rate from the Deconfined Phase,” Phys. Rev. C 83, 014908 (2011) [arXiv:1010.2169 [hep-ph]].

[17] M. G. Mustafa, A. Schafer and M. H. Thoma, "Nonperturbative dilepton production from a quark gluon plasma,” Phys. Rev. C 61, 024902 (2000) [hep-ph/9908461].

[18] S. P. Klevansky, “The Nambu-Jona-Lasinio model of quantum chromodynamics," Rev. Mod. Phys. 64, 649 (1992).

[19] M. Buballa, "NJL model analysis of quark matter at large density,” Phys. Rept. 407, 205 (2005) [hep-ph/0402234].

[20] F. Karsch, M. G. Mustafa and M. H. Thoma, "Finite temperature meson correlation functions in HTL approximation,” Phys. Lett. B 497, 249 (2001) [hep-ph/0007093].

[21] C. A. Islam, S. Majumder, N. Haque and M. G. Mustafa, "Vector meson spectral function and dilepton production rate in a hot and dense medium within an effective QCD approach,” JHEP 1502, 011 (2015) [arXiv:1411.6407 [hep-ph]]. 